\title{
Children with extreme hyperferritinemia are at risk of receiving more chemotherapy than necessary
}

\author{
Seung Beom Han, Soo-Young Lee ${ }^{\odot}$ \\ Department of Pediatrics, Bucheon St. Mary's Hospital, College of Medicine, The Catholic University of Korea, Seoul, Republic of Korea
}

\section{Dear Editor,}

We read with interest the article by Çakan et al. ${ }^{1}$ and wish to add our own experience. The authors reported a 9-year-old boy with macrophage activation syndrome (MAS) secondary to systemic juvenile idiopathic arthritis (sJIA). We agree with their opinion that genetic testing for familial hemophagocytic lymphohistiocytosis (HLH) should be performed when a patient with MAS exhibits sustained hyperferritinemia. However, there is another important lesson when the patient exhibits extreme hyperferritinemia.

A 16-month-old girl was referred from a general hospital to our hospital with a 10day fever. On physical examination, there were no abnormalities except for palpable

Soo-Young Lee

sylee@catholic.ac.kr

Received 15th May 2019; accepted 27th September 2019
$3 \mathrm{~cm}$ liver and $5-\mathrm{cm}$ spleen below the costal margin. Blood tests showed increased acute phase reactants and elevated liver enzymes. Considering the possibility of serious bacterial infection and sepsis, empiric antibiotics (cefotaxime $200 \mathrm{mg} / \mathrm{kg} /$ day) and intravenous immunoglobulin (400 mg/kg for 5 days) were administered. ${ }^{2}$ However, her clinical and laboratory findings became aggravated and met the HLH diagnostic criteria (Table I). ${ }^{3}$ She was transferred to pediatric hematology team and received 40 weeks of chemotherapy including etoposide $\left(150 \mathrm{mg} / \mathrm{m}^{2}\right.$; i.e., the HLH-2004 protocol). She did not show any relapse of the disease during therapy and entered remission when she was 28 months old. Two months after terminating therapy, she visited the hospital again with fever, rash, and arthritis. Only then, was she eventually diagnosed with sJIA with the presenting manifestation of MAS. She now receives maintenance therapy for sJIA and has experienced no further relapse.

Table I. Laboratory findings of the patient.

\begin{tabular}{lcccc}
\hline Parameters & $1^{\text {st }}$ day & $8^{\text {th }}$ day & $3^{\text {th }}$ day & 1 year later \\
\hline Leukocytes, $/ \mathrm{mm}^{3}$ & 15,700 & 3,800 & 5,000 & 5,700 \\
Hemoglobin, g/dl & 11.6 & 6.4 & 11.2 & 13.6 \\
Platelet, /mm & 188,000 & 51,000 & 432,000 & 272,000 \\
CRP, mg/L & 184 & 56 & 0.4 & 0.2 \\
ESR, mm/hour & 56 & 28 & 12 & 4 \\
AST, IU/L & 199 & 1,006 & 22 & 25 \\
ALT, IU/L & 109 & 397 & 17 & 24 \\
Ferritin, ng/ml & 3,730 & 97,100 & 1,500 & 43 \\
Triglyceride, mg/dl & - & 288 & 107 & 87 \\
Fibrinogen, mg/dl & - & 139 & 197 & 280 \\
LDH, U/L & - & 2,131 & 823 & 392
\end{tabular}

ALT: alanine aminotransferase, AST: aspartate aminotransferase, CRP: C-reactive protein, ESR: erythrocyte sedimentation rate, $\mathrm{LDH}$; lactate dehydrogenase. 
Reviewing her medical records in detail, we noted that 40 weeks of chemotherapy including etoposide might not have been necessary for disease management. Children with MAS complicating sJIA can have good outcomes with short-term immunomodulators (i.e., the 8-week steroids and cyclosporine). ${ }^{4}$ However, when children with unexplained MAS exhibit extreme hyperferritinemia (ferritin: 100,000 ng/ $\mathrm{ml}){ }^{1,5}$ they are at risk of receiving long-term potentially toxic chemotherapy because their overwhelming clinical manifestations usually meet the HLH diagnostic criteria. ${ }^{6}$ MAS is a serious, life-threatening complication of childhood systemic inflammatory disorders. ${ }^{1,3}$ Therefore, prompt initiation of adequate treatment is essential for the survival of affected children. ${ }^{4-6}$ At the same time, careful monitoring of therapeutic response is also necessary to avoid overtreatment of MAS.

\section{REFERENCES}

1. Çakan M, Aktay-Ayaz N, Gemici H, et al. Sustained hyperferritinemia in a child with macrophage activation syndrome secondary to systemic juvenile idiopathic arthritis-perforinopathy: case based review. Turk J Pediatr 2018; 60: 598-603.

2. Alejandria MM, Lansang MA, Dans LF, Mantaring JB 3rd. Intravenous immunoglobulin for treating sepsis, severe sepsis and septic shock. Cochrane Database Syst Rev 2013; CD001090.

3. Henter JI, Horne AC, Aricó M, et al. HLH2004: diagnostic and therapeutic guidelines for hemophagocytic lymphohistiocytosis. Pediatr Blood Cancer 2007; 48: 124-131.

4. Demirkol D, Yildizdas D, Bayrakci B, et al; Turkish Secondary HLH/MAS Critical Care Study Group. Hyperferritinemia in the critically ill child with secondary hemophagocytic lymphohistiocytosis/ sepsis/multiple organ dysfunction syndrome/ macrophage activation syndrome: what is the treatment? Crit Care 2012; 16: R52.

5. Avcin T, Tse SM, Schneider R, Ngan B, Silverman ED. Macrophage activation syndrome as the presenting manifestation of rheumatic diseases in childhood. J Pediatr 2006; 148: 683-686.

6. Haytoglu Z, Yazici N, Erbay A. Secondary hemophagocytic lymphohistiocytosis: do we really need chemotherapeutics for all patients? J Pediatr Hematol Oncol 2017; 39: e106-e109. 